\title{
Osteoradionecrosis of Jaw in Head and Neck Cancer Patient Treated with Free lliac Bone and Umbilical Fat Pad Graft
}

\author{
Yuri Choi, Su-Gwan Kim, Seong-Yong Moon, Ji-Su Oh, Jae-Seek You, \\ Kyung-In Jeong, Sung-Seok Lee
}

Department of Oral and Maxillofacial Surgery, School of Dentistry, Chosun University

\begin{abstract}
Osteoradionecrosis is one of the most serious complications of patients receiving radiation therapy. It is characterized by hypovascularity, hypocellularity, and hypoxia-inducing necrosis of bone and soft tissue following delayed healing. In this case, a 72-year-old man was referred to the Department of Oral and Maxillofacial Surgery complaining of trismus following extraction three months before first visit. He had a history of right tonsillectomy, radical neck dissection and radiotherapy performed due to right tonsillar cancer seven years prior. After the diagnosis of osteoradionecrosis on right mandibular body and angle, conservative antibiotic therapy was used first, but an orocutaneous fistula gradually formed, and extensive bony destruction and sequestrum were observed. Sequestrectomy, free particulated iliac bone and umbilical fat pad graft were performed via a submandibular approach under general anesthesia. Preoperative regular exams and delicate wound care led to secondary healing of the wound without vascularized free flap reconstruction.
\end{abstract}

Key words: Osteoradionecrosis, Head and neck neoplasms, Radiotherapy

\section{Introduction}

Radiation therapy is a standard treatment for head and neck malignancy. Radiation therapy targets cells that have a rapid cell cycle regardless of malignancy. Thus it becomes very important to balance conservation of normal cells and elimination of affected cells. Sometimes limitation of duration and quantity of radiation therapy is necessary to minimize side effects on normal tissue, and in severe cases alternative treatment is considered. Osteoradionecrosis is one of the most serious complications of radiation therapy. Treatment is difficult, and often osteoradionecrosis leads to damage such as deformity of jaws. Several theories for the pathogenesis of osteonecrosis have been suggested, and it is widely accepted that hypoxic, hypovascular and hypocellular change of radiation exposed bone are a primary mechanism[1].

Osteoradionecrosis is defined as non-vital bone appearing at irradiated areas, and is characterized by delayed healing with little new bone formation locally for 3 to 6

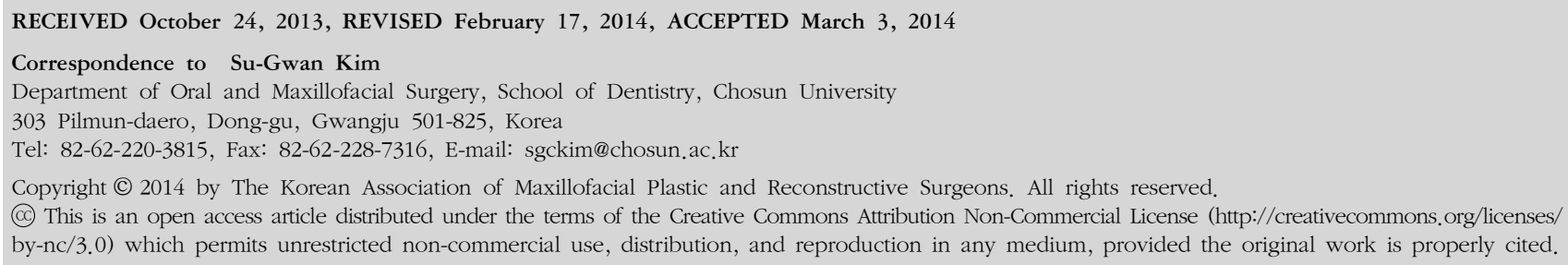


months. This condition affects not only bone but also nearby soft tissue[2]. Morbidity of the mandible is higher than that of the maxilla. The mandibular body is the most affected site, with over $50 \%$ of cases occurring after tooth extraction[3].

As head and neck cancer patients are often dentulous, preliminary preventive dental treatment is very important. Although several treatments for osteoradionecrosis exist, such as local wound debridement, antibiotics, surgical treatment and hyperbaric oxygen therapy, definitive treat-

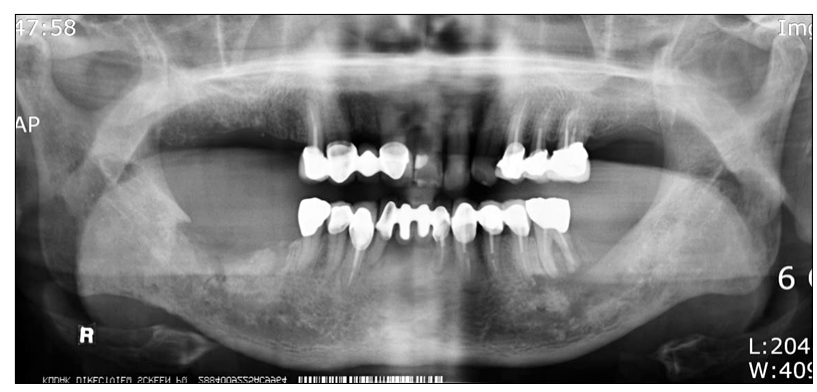

Fig. 1. Panoramic view on first visit: 3 months after extraction of \#46, 47. ment for osteoradionecrosis is controversial.

This paper is a case report of osteoradionecrosis of the mandible that was treated with sequestrectomy and iliac bone graft, with a current literature review.

\section{Case Report}

A 72-year-old male patient visited the Department of Oral Medicine with trismus following extraction. The patient's medical history includes a tonsillectomy and right radical neck dissection followed by radiation therapy due to right tonsillar cancer seven years prior. Three months before the visit, the right mandibular first molar and second molar were extracted at a local dental clinic, followed by trismus developing. At the first visit, the mouth opening was $10 \mathrm{~mm}$, nerve damage signs were absent, and a moth-eaten appearance was observed at the right mandibular angle area in panoramic view (Fig. 1). Osteomyelitis was diagnosed and he was referred to the Department of Oral and Maxillofacial Surgery. He was treated conservatively with antibiotics. After eight months, an in-
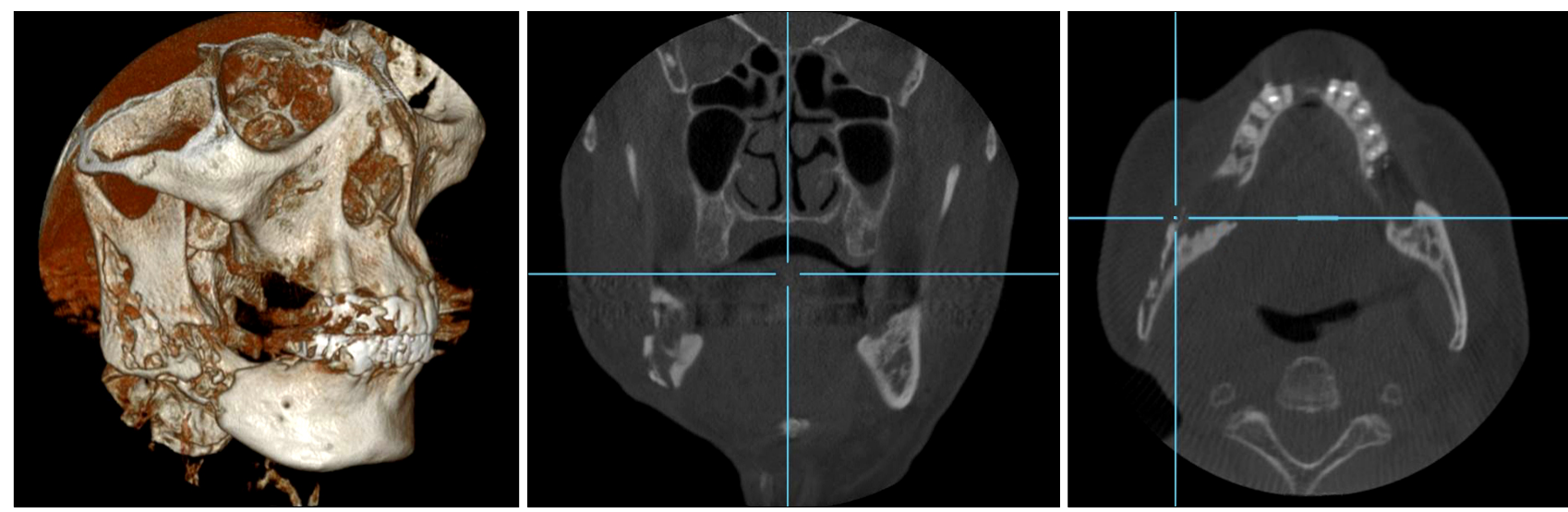

Fig. 2. Cone-beam computed tomographic view before operation.
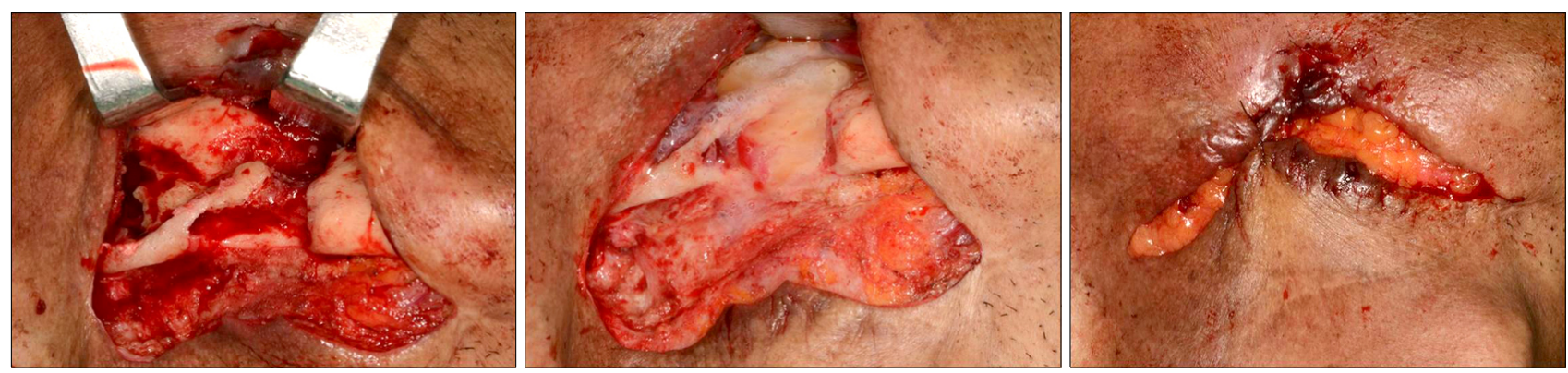

Fig. 3. Sequestrectomy, left iliac bone and umbilical fat pad graft was done under general anesthesia via submandibular approach. 
duration manifested at the right mandibular angle and submandibular area; an orocutaneous fistula formed, with pus discharge above the right mandibular inferior border area and right mandibular posterior edentulous gingiva. From cone-beam computed tomography (CT) and panoramic view, extensive bone destruction was observed from the right mandibular body to the mandibular angle area. Sequestrum was also observed (Fig. 2). Sequestrectomy, iliac bone graft, and umbilical fat pad graft was performed via a submandibular approach under general anesthesia (Fig. 3). One month after the operation wound dehiscence was observed at the submandibular operation site. To promote secondary healing, Terudermis (Olympus Terumo Biomaterials Corp., Tokyo, Japan) was applied to the wound dehiscence (Fig. 4). As secondary healing proceeded, wound dehiscence recovered, there was no more pus discharge, and mouth opening improved to over 30 mm (Fig. 5, 6).

\section{Discussion}

Radiation therapy and dental treatment development have decreased the risk of osteoradionecrosis of the jaw. Still, osteoradionecrosis remains the most severe complication of radiation therapy[4]. Since there is no exact definition of osteoradionecrosis, diagnosis depends on clinical symptoms, with radiographic findings also suggested as diagnostic criteria. Typical symptoms of osteoradionecrosis include nonvital bone exposure, sequestrum, orocutaneous fistula and pathologic fracture of mandible[2]. The bone exposure period is highly controversial as a diagnosis criterion. Generally osteoradionecrosis is diagnosed if non-vital bone exposure with delayed healing lasts three to six months, with non-specific neoplastic findings of the
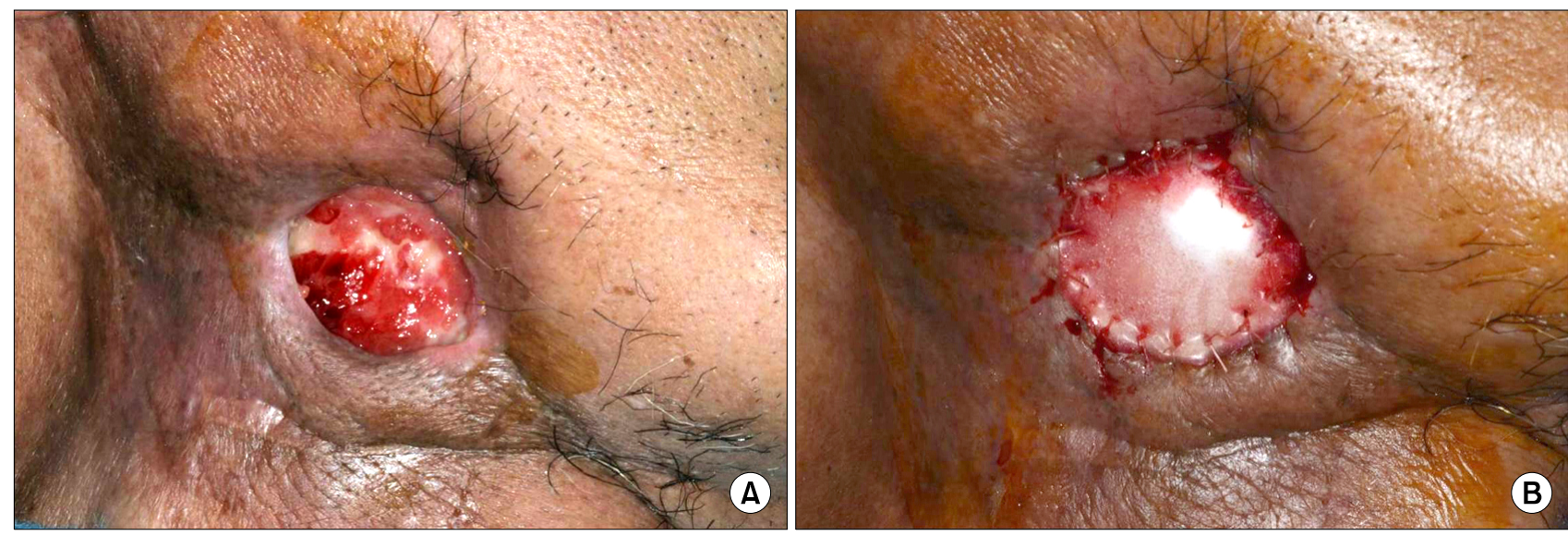

Fig. 4. (A) Wound dehiscence on submandibular operation site 1 month after operation. (B) Terudermis (Olympus Terumo Biomaterials Corp., Tokyo, Japan) was applied to promote secondary wound healing.

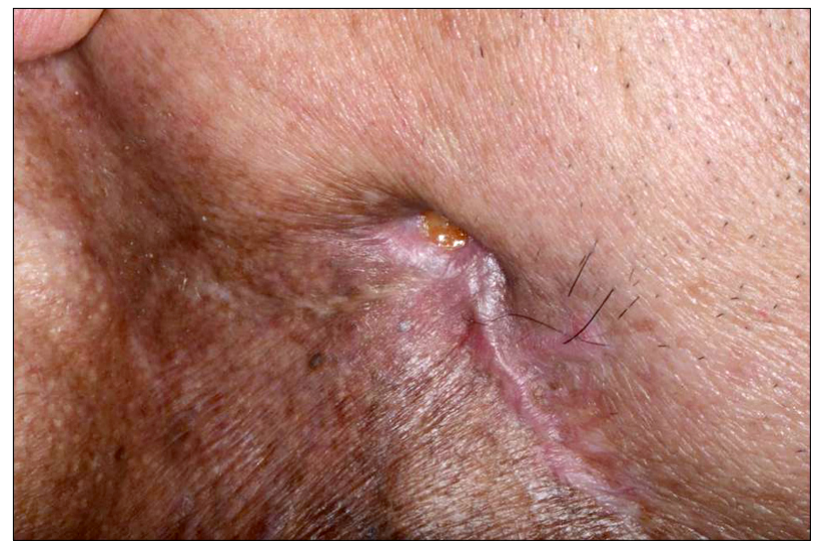

Fig. 5. Dehiscence was closed with secondary wound healing.

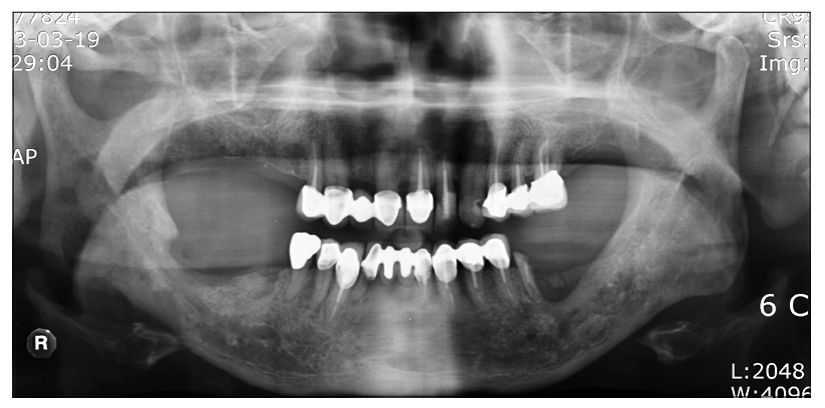

Fig. 6. Panoramic view on postoperative exam 8 months after surgery. 
bone[5].

Tooth extraction is the main risk factor for osteoradionecrosis of jaw. The exact morbidity rate following tooth extraction is not known but several reports state that about $2 \%$ of tooth extraction leads to osteoradionecrosis. The mandible has higher morbidity than the maxilla. The difference in morbidity rate is thought to stem from the greater radiation exposure to the mandible, and the effect on blood supply during radical neck dissection, cutting facial artery and branches[6].

Before the 1990s, morbidity of almost 16\% was reported, but radiation technique development reduced that to $2 \%[2,7]$. In addition to development of radiation technique, part of the decreasing risk of osteoradionecrosis is attributed to less frequent teeth extraction after radiation therapy, due to increased dental care and caries prevention with fluoride for head and neck cancer patients.

When osteoradionecrosis is suspected after tooth extraction, delayed healing of less than three months is difficult to diagnose as osteoradionecrosis, because radiation exposed tissue has low recovery capacity. In that situation, observation is recommended over surgical treatment. Once osteoradionecrosis is diagnosed, treatment depends on the affected range of the lesion. In localized and stabilized osteoradionecrosis, conservative therapy such as hyperbaric oxygen therapy, careful oral hygiene management and local sequestrectomy is preferred, accompanied by long-term antibiotic therapy. However, if the patient is suffering from pain, if there is bone exposure, if there is lack of response to antibiotics, trismus, oroskin fistula or pathologic fracture, an extensive surgical approach is necessary[8].

The basic principle of the surgical approach for osteoradionecrosis is elimination of all necrotic tissue and severe scar tissue, and reconstruction of the defect with well-vascularized tissue[8,9]. As in extensive osteonecrosis, segmental mandiblectomy and soft tissue excision is often necessary. At the same time, vascularized free flap reconstruction is also considerable. Non-vascularized free bone graft into a defect has a limited success rate[10,11], and especially with radiation wounds, a high complication rate[12]. A well-vascularized free flap yields more predictable and esthetic results compared with non-vascularized tissue graft[13]. An abundant blood supply in the non-radia- tion-exposed vascularized graft tissue can promote healing even in osteoradionecrosis affected marginal tissue. Localized flap or distant flap can also provide vascularized tissue but these flaps are less reliable in providing sufficient blood supply comparing with a vascularized free flap[14]. In general, microvascular reconstruction is thought to be the most effective reconstruction method for advanced osteoradionecrosis. The greatest advantage of microvascular reconstruction is safety resulting from intrinsic blood supply from a main perforator vessel even at an extensive lesion. Furthermore, this can supply muscle or skin complex simultaneously.

This study presents osteoradionecrosis on a recurrent tonsil cancer patient who had repeated radiation therapy and radical neck dissection. Soundness of the lingual cortex was checked on the cone-beam CT images, and the lingual cortex was identified after sequestrectomy. During surgery, normal facial artery and branches were not detected, probably a result of repeated radiotherapy and radical neck dissection. Thus, sequestrectomy and non-vascularized iliac bone graft were selected as the alternative treatment methods for osteoradionecrosis of the mandible. At postoperative exam, although there was noticeable delay in the healing process, especially wound dehiscence, regular wound dressing and secondary healing process using Terudermis (Olympus Terumo Biomaterials Corp.) coverage gave satisfactory results.

At this point the patient has no more pain and pus drainage and neither the necrosis nor the cancer have recurred. With extensive osteoradionecrosis, mandibular reconstruction using vascularized free flap should be considered first. In this case, with a localized osteoradionecrosis lesion where preoperative conservative therapy was sufficient, satisfactory results were obtained using free iliac bone and an umbilical fat pad graft with an abundant blood supply.

\section{References}

1. Harris M. The conservative management of osteoradionecrosis of the mandible with ultrasound therapy. Br J Oral Maxillofac Surg 1992;30:313-8.

2. Beumer J, Harrison R, Sanders B, Kurrasch M. Osteoradionecrosis: predisposing factors and outcomes of therapy. Head Neck Surg 1984;6:819-27.

3. Reuther T, Schuster T, Mende U, Kübler A. Osteoradionecrosis of the jaws as a side effect of radiotherapy of head and 
neck tumour patients--a report of a thirty year retrospective review. Int J Oral Maxillofac Surg 2003;32:289-95.

4. Friedman RB. Osteoradionecrosis: causes and prevention. NCI Monogr 1990;(9):145-9.

5. Støre G, Boysen M. Mandibular osteoradionecrosis: clinical behaviour and diagnostic aspects. Clin Otolaryngol Allied Sci 2000;25:378-84.

6. Beumer J 3rd, Harrison R, Sanders B, Kurrasch M. Postradiation dental extractions: a review of the literature and a report of 72 episodes. Head Neck Surg 1983;6:581-6.

7. Curi MM, Dib LL. Osteoradionecrosis of the jaws: a retrospective study of the background factors and treatment in 104 cases. J Oral Maxillofac Surg 1997;55:540-4.

8. Nakatsuka T, Harii K, Yamada A, Ueda K, Ebihara S, Takato $\mathrm{T}$. Surgical treatment of mandibular osteoradionecrosis: versatility of the scapular osteocutaneous flap. Scand J Plast Reconstr Surg Hand Surg 1996;30:291-8.
9. Obwegeser HL, Sailer HF. Experience with intraoral resection and immediate reconstruction in cases of radio-osteomyelitis of the mandible. J Maxillofac Surg 1978;6:257-65.

10. Robinson DW. Surgical problems in the excision and repair of radiated tissue. Plast Reconstr Surg 1975;55:41-9.

11. Duncan MJ, Manktelow RT, Zuker RM, Rosen IB. Mandibular reconstruction in the radiated patient: the role of osteocutaneous free tissue transfers. Plast Reconstr Surg 1985;76:829-40.

12. Adamo AK, Szal RL. Timing, results, and complications of mandibular reconstructive surgery: report of 32 cases. J Oral Surg 1979;37:755-63.

13. Lydiatt DD, Lydiatt WM, Hollins RR, Friedman A. Use of free fibula flap in patients with prior failed mandibular reconstruction. J Oral Maxillofac Surg 1998;56:444-6.

14. Baker SR. Management of osteoradionecrosis of the mandible with myocutaneous flaps. J Surg Oncol 1983;24:282-9. 